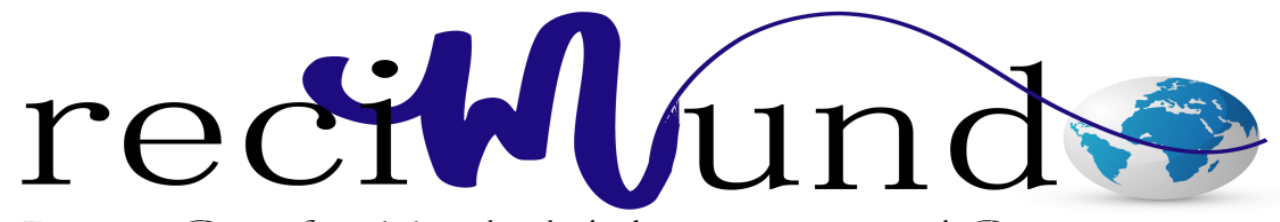

Revista Científica Mundo de la Investigación y el Conocimiento

Elio Edwin Sánchez Suárez ${ }^{\text {a; }}$ Benita del Carmen Cañizalez de Aguirre ${ }^{b}$

Activos Financieros administrados por el Instituto Ecuatoriano de Seguridad Social

Revista Científica Mundo de la Investigación y el Conocimiento. Vol. 2 núm., 1, febrero, ISSN: 2588-073X, 2018, pp. 374-392

DOI: 10.26820/recimundo/2.1.2018.374-392

Editorial Saberes del Conocimiento

Recibido: 05/12/2017

Aceptado: 10/02/2018

a. Universidad de Guayaquil; elio.sanchezs@ug.edu.ec

b. Universidad de Guayaquil; benita.canizalezd@ug.edu.ec 


\section{Activos Financieros administrados por el Instituto Ecuatoriano de Seguridad}

Social

Vol. 2, núm. 1., (2018)

Elio Edwin Sánchez Suárez; Benita del Carmen Cañizalez de Aguirre

\section{RESUMEN}

La historia financiera de América Latina padece la influencia del desarrollo económico del resto del mundo. Pareciera ser el destino de estos países sobrevivir a las sombras de un mundo cada vez más pesado de sobrellevar cuando se trata de superación y subsistencia. Durante el gobierno de Rafael Correa se toma la iniciativa de propiciar planes y proyectos integrados que garanticen el éxito del desarrollo equitativo de la población. Las Actividades Financieras, son un servicio de orden público, y podrán ejercerse, previa autorización del Estado, de acuerdo con la ley. La Constitución define al Instituto Ecuatoriano de Seguridad Social (IESS) como el ente responsable de la prestación de las contingencias del seguro universal obligatorio de los afiliados; y, la Ley de Seguridad Social, se extiende y define que el IESS es una entidad pública descentralizada dotada de autonomía normativa, técnica, administrativa, financiera y presupuestaria, con personería jurídica y patrimonio propio, que tiene por objeto indelegable la prestación del Seguro General Obligatorio en todo el territorio ecuatoriano. Esta investigación es exploratoria, experimental de corte transversal para hacer un análisis del comportamiento económico de los activos financieros en los que puede invertir la administración del IESS y en efecto en los que ha invertido durante el periodo 2007-2012. La intervención del IESS en el sector Financiero ecuatoriano ha sido positiva, sobre todo a través de operaciones bursátiles en el mercado de valores, canalizando los recursos financiaros de los agentes excedentarios a actividades productivas materializadas y promovidas por agentes deficitarios de estos mismos recursos financieros, produciendo un efecto multiplicador en la economía ecuatoriana que atrae sistemáticamente mayor inversión tanto pública como privada.

Palabras clave: Seguridad, social, inversión, financiero, mercados. 


\title{
Activos Financieros administrados por el Instituto Ecuatoriano de Seguridad Social
}

Vol. 2, núm. 1., (2018)

Elio Edwin Sánchez Suárez; Benita del Carmen Cañizalez de Aguirre

\begin{abstract}
The financial history of Latin America suffers from the influence of the economic development of the rest of the world. It seems to be the destiny of these countries to survive the shadows of an increasingly heavy world to endure when it comes to overcoming and subsistence. During the government of Rafael Correa, the initiative is taken to promote plans and integrated projects that guarantee the success of the equitable development of the population. The Financial Activities are a public order service, and may be exercised, with the prior authorization of the State, in accordance with the law. The Constitution defines the Ecuadorian Institute of Social Security (IESS) as the entity responsible for the provision of contingencies of universal compulsory insurance for members; and, the Social Security Law, extends and defines that the IESS is a decentralized public entity endowed with normative, technical, administrative, financial and budgetary autonomy, with legal status and its own assets, whose object is non-delegable the provision of General Insurance Obligatory in the entire Ecuadorian territory. This research is exploratory, cross-sectional experimental to make an analysis of the economic behavior of the financial assets in which the administration of the IESS can invest and indeed in those that have invested during the period 2007-2012. The intervention of the IESS in the Ecuadorian financial sector has been positive, especially through stock market operations, channeling the financial resources of the surplus agents to productive activities materialized and promoted by deficient agents of these same financial resources, producing a multiplier effect in the Ecuadorian economy that systematically attracts greater public and private investment.
\end{abstract}

Keywords: Security, social, investment, financial, markets. 


\section{Activos Financieros administrados por el Instituto Ecuatoriano de Seguridad}

Social

Vol. 2, núm. 1., (2018)

Elio Edwin Sánchez Suárez; Benita del Carmen Cañizalez de Aguirre

\section{Introducción.}

La historia financiera de América Latina padece la influencia del desarrollo económico del resto del mundo. Pareciera ser el destino de estos países sobrevivir a las sombras de un mundo cada vez más pesado de sobrellevar cuando se trata de superación y subsistencia.

Ecuador no está exento de esta realidad. En la historia contemporánea de la región grandes cambios se han evidenciado, sobretodo en el ámbito político que repercute directamente en las políticas económicas imperantes en la región.

Durante el gobierno de Rafael Correa se plantearon una cantidad de reformas en todos los frentes de trabajo de la sociedad en la búsqueda de potenciar el desarrollo de la sociedad ecuatoriana. Desde el gobierno central se toma la iniciativa de propiciar planes y proyectos integrados que garanticen el éxito del desarrollo equitativo de la población.

Uno de los frentes de estos cambios se hace evidente en los proyectos en la estructura económica del país. Desde las reformas constitucionales hasta los proyectos educativos reflejan el ánimo de éstos cambios.

Las Actividades Financieras, son un servicio de orden público, y podrán ejercerse, previa autorización del Estado, de acuerdo con la ley y normativas vigentes tendrán la finalidad fundamental de preservar los depósitos y atender los requerimientos de financiamiento para la consecución de los objetivos de desarrollo del país. Las actividades financieras intermediarán de forma eficiente los recursos captados del público para fortalecer la inversión productiva nacional, y el consumo social y ambientalmente responsable. 


\section{Activos Financieros administrados por el Instituto Ecuatoriano de Seguridad Social}

Vol. 2, núm. 1., (2018)

Elio Edwin Sánchez Suárez; Benita del Carmen Cañizalez de Aguirre

La concesión de préstamos y créditos depende de la cantidad de recursos disponibles, es decir, del ahorro de los usuarios que han de servir para transformarlos en fuentes de financiamiento. La asignación de recursos procura la distribución de los recursos disponibles en una economía para obtener la máxima productividad posible.

La creación de liquidez supone el efectivo en circulación en una economía, el resto del dinero lo crean las entidades financieras con la concesión de préstamos y créditos.

- En resumen, las principales funciones del sistema financiero son:

- Captar el ahorro y canalizarlo hacia la inversión productiva.

- Ofertar productos financieros.

- Lograr la estabilidad monetaria.

- Proporcionar mecanismos que canalicen los flujos financieros.

- Mantener el equilibrio financiero en el economía.

- Valorar el riesgo.

- Gestionar medios de pago.

- Contribuir a la estabilidad del sistema monetario.

La seguridad social, tal como se enuncia por la Constitución de la Organización Internacional del Trabajo (OIT), es un instrumento fundamental para el progreso y el desarrollo de los pueblos. Su promoción y extensión se enmarca en los objetivos estratégicos de la OIT.

La Seguridad Social es un término que se refiere al estado de bienestar. Una definición armonizada y que encaja para todas las economías, la definió OIT (1991) en un documento 


\section{Activos Financieros administrados por el Instituto Ecuatoriano de Seguridad}

Social

Vol. 2, núm. 1., (2018)

Elio Edwin Sánchez Suárez; Benita del Carmen Cañizalez de Aguirre

denominado "Administración de la Seguridad Social" como: La protección que la sociedad proporciona a sus miembros, mediante una serie de medidas públicas, contra las privaciones económicas y sociales que, de no ser así, ocasionarían la desaparición o una fuerte reducción de los ingresos por causa de enfermedad, maternidad, accidente de trabajo, o enfermedad de tipo laboral, desempleo o paro, invalidez, vejez y muerte; y asistencia médica y ayuda a las familias con hijos. El sistema de seguridad social participa en los mercados financieros locales, porque realiza transacciones financieras en una economía, mediante instrumentos que permiten la transferencia de fondos temporalmente libres entre entes económicos y agentes económicos.

\section{Sistema Nacional de Seguridad Social}

El sistema nacional de seguridad social (SNSS), corresponde a la dotación de programas de seguros complementarios de propiedad privada, pública o mixta. En la siguiente ilustración presentamos a las instituciones:

\section{Cuadro 1: Sistema Nacional de Seguridad Social}

\begin{tabular}{l}
\hline Entidades que integran: \\
Instituto Ecuatoriano de Seguridad Social \\
(IESS). \\
Instituto de Seguridad Social de las \\
Fuerzas Armadas (ISSFA). \\
Instituto de Seguridad Social de la \\
Policía Nacional (ISSPOL). \\
Unidades Médicas Prestadoras de Salud \\
(UMPS) \\
Personas jurídicas que administran \\
programas de seguros complementarios \\
(publico, privado y mixto).
\end{tabular}

Fuente: Ley de Seguridad Social 


\section{Activos Financieros administrados por el Instituto Ecuatoriano de Seguridad Social}

Vol. 2, núm. 1., (2018)

Elio Edwin Sánchez Suárez; Benita del Carmen Cañizalez de Aguirre

Las entidades que integran el Sistema Nacional de Seguridad Social ecuatoriano y las personas naturales y jurídicas que integran el Sistema de Seguro Privado, para su constitución, organización, actividades, funcionamiento y extinción se sujetarán estrictamente a las disposiciones de la Ley de Seguridad Social, a la Ley General de Seguros y su Reglamento, a la Ley General de Instituciones del Sistema Financiero y su Reglamento, a la Ley Orgánica de Administración Financiera y Control, a la Ley de Mercado de Valores, al Código de Comercio, a la Ley de Compañías y a las normas reglamentarias y resoluciones que dicten los organismos de control creados por la Constitución del Ecuador.

El Seguro General Obligatorio ss parte del Sistema Nacional de Seguridad Social y los afiliados son sujetos "obligados a solicitar la protección" todo aquel que perciben ingresos por la ejecución de una obra o la prestación de un servicio físico o intelectual, con relación laboral o sin ella.

EI seguro universal obligatorio cubre las contingencias de enfermedad, maternidad, paternidad, riesgos de trabajo, cesantía, desempleo, vejez, invalidez, discapacidad, muerte y aquellas que sean definidas por la ley.

La Constitución del Ecuador y los instrumentos internacionales, garantizan sin discriminación alguna el efectivo goce de los derechos, en particular la educación, la salud, la alimentación y la seguridad social.

La sección tercera del Régimen del Buen Vivir de la Constitución indica que el Sistema de Seguridad Social comprenderá a las entidades públicas, normas, políticas, recursos, servicios 


\section{Activos Financieros administrados por el Instituto Ecuatoriano de Seguridad}

Social

Vol. 2, núm. 1., (2018)

Elio Edwin Sánchez Suárez; Benita del Carmen Cañizalez de Aguirre

y prestaciones de seguridad social, y funcionará con base en los criterios de sostenibilidad, eficiencia, celeridad y transparencia

La Constitución define al IESS como el ente responsable de la prestación de las contingencias del seguro universal obligatorio de los afiliados; y, la Ley de Seguridad Social, se extiende y define que el IESS es una entidad pública descentralizada, creada por la Constitución Política de la República del Ecuador, dotada de autonomía normativa, técnica, administrativa, financiera y presupuestaria, con personería jurídica y patrimonio propio, que tiene por objeto indelegable la prestación del Seguro General Obligatorio en todo el territorio ecuatoriano.

Conociendo las tareas a desarrollar por el Sistema de seguridad Social nos proponemos en la siguiente investigación describir los activos financieros que se manejan desde el Instituto ecuatoriano de Seguridad Social como fuente generadora de riquezas para la población.

\section{Materiales y métodos.}

Esta investigación es exploratoria, experimental de corte transversal pues se toma como fuente de información, no solo la normativa referente al caso de estudio para hacer un esquema de la operatividad y funcionabilidad del Instituto Ecuatoriano de Seguridad Social, sino que se revisan datos publicados por el Banco Central del Ecuador para hacer un análisis del comportamiento económico de los activos financieros en los que puede invertir la administración del IESS y en efecto en los que ha invertido durante el periodo 2007-2012 


\section{Activos Financieros administrados por el Instituto Ecuatoriano de Seguridad Social}

Vol. 2, núm. 1., (2018)

Elio Edwin Sánchez Suárez; Benita del Carmen Cañizalez de Aguirre

\section{Resultados.}

\section{La ley de Seguridad Social}

El Seguro General Obligatorio forma parte del sistema nacional de seguridad social y, como tal, su organización y funcionamiento se fundamentan en los principios de solidaridad, obligatoriedad, universalidad, equidad, eficiencia, subsidiariedad y suficiencia.8 La Ley reformatoria de la Ley de Seguridad Social beneficia a 276.408 pensionistas del IESS, que incrementó sus pensiones entre 40 y 60 dólares en el 2010. Los 276.498 pensionistas están considerados en cuatro grupos: 17.704 por invalidez; 168.790 por vejez; 56.672 por viudedad; 33.242 por orfandad. A partir del 2011 las pensiones se incrementan automáticamente por medio de cinco coeficientes, con lo cual quienes menos reciben, tendrán un incremento mayor. Para las viudas sus pensiones se incrementan del 40 a $60 \%$ en relación con la renta de su cónyuge o conviviente reconocido. Los hijos e hijas de los afiliados y pensionistas recibirán servicios gratuitos de salud hasta los 18 años de edad y los viudos de las afiliadas tienen derecho a recibir sus pensiones.

Estructura de las Inversiones Del IESS en el Mercado de Valores.

La Banca de Inversión es la institución especializada que tiene como objeto principal intervenir en la colocación de capitales a través de la búsqueda y obtención de financiamiento para proyectos de inversión (inversiones a largo plazo) a través del mercado de valores.

En este sentido, el objeto social del Banco del Instituto Ecuatoriano de Seguridad Social (BIESS) es la prestación de servicios financieros bajo criterios de banca de inversión, para la 


\section{Activos Financieros administrados por el Instituto Ecuatoriano de Seguridad}

Social

Vol. 2, núm. 1., (2018)

Elio Edwin Sánchez Suárez; Benita del Carmen Cañizalez de Aguirre

administración de los fondos previsionales 12 públicos del Instituto Ecuatoriano de Seguridad Social (IESS); y, la prestación de servicios financieros, para atender los requerimientos de sus afiliados activos y jubilados.

Tabla 1: Operaciones financieras

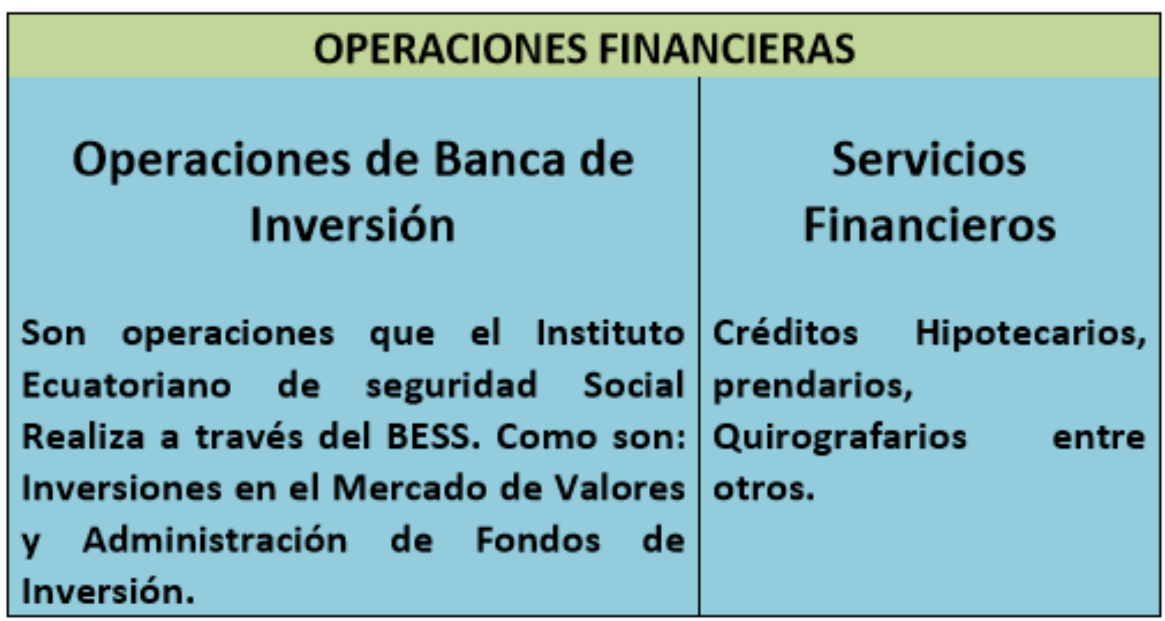

Fuente: Banco del Instituto de Seguridad Social (BIESS)

Las inversiones de recursos previsionales están destinadas a la concesión de créditos a los afiliados, sector real, sector público, sector financiero y sectores estratégicos de la economía.

Las operaciones de banca de inversión son las siguientes:

-Inversiones a través de los instrumentos que ofrece el mercado de valores para el financiamiento a largo plazo de proyectos públicos y privados, productivos y de infraestructura.

-Inversiones en el mercado de valores en títulos de renta fija (bonos) o variable a través del mercado primario y secundario. 


\section{Activos Financieros administrados por el Instituto Ecuatoriano de Seguridad Social}

Vol. 2, núm. 1., (2018)

Elio Edwin Sánchez Suárez; Benita del Carmen Cañizalez de Aguirre

-Estructurar, impulsar y promover proyectos de inversión.

Los servicios financieros son los siguientes:

-Conceder créditos hipotecarios, prendarios y quirografarios y otros servicios financieros a favor de los afiliados y jubilados del IESS, mediante operaciones directas o a través del sistema financiero nacional.

-Operaciones de redescuento de cartera hipotecaria de instituciones financieras.

El BIESS a más de lo establecido, podrá realizar las siguientes operaciones:

-Estructuración de portafolios de valores; negociación de paquetes accionarios, adquisiciones, fusiones; $y$,

-Participar como constituyente o adherente beneficiario en negocios fiduciarios, mercantiles, administración, gestión, inmobiliarios, inversión o mixtos. Los servicios de Banca de Inversión16 del BIESS se clasifican en: 


\section{Activos Financieros administrados por el Instituto Ecuatoriano de Seguridad}

Social

Vol. 2, núm. 1., (2018)

Elio Edwin Sánchez Suárez; Benita del Carmen Cañizalez de Aguirre

Tabla 2: Productos financieros

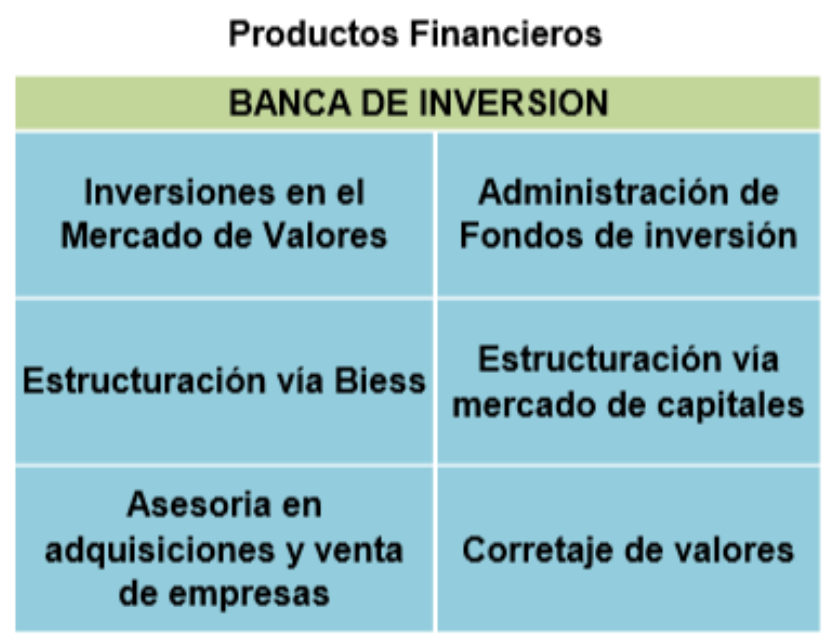

Fuente: Banco del IESS

El IESS ha invertidito en títulos de grandes compañías como Nestlé, Diners Club, Promérica, Automotores y Anexos, KFC, Pacificard, De Prati, La Fabril, entre otras. También mantiene papeles de firmas como Tía, Telconet, Industrias Ales, Corporación El Rosado, Industria Licorera Ecuatoriana, Pronaca, Incable, Pronaca, La Tablita, Adfranec, Eslive, Mamut Andino, Titularización Sindicada - Equitatis Ibc, Constructora E Inmobiliaria Valero, Reporto Corporación Favorita, Titularización Sindicada, Equitatis Ibc, Ecogal, Plasticos Del Litoral, Tercera Tit.Cartera Comercial - Iiasa, Ministerio De Finanzas, La Fabril, entre otros.

Volumen de Negociaciones de Valores El Volumen de operaciones, según las estadísticas del Banco Central del Ecuador (BCE), contiene el volumen de transacciones de papeles fiduciarios negociados en la Bolsa de Valores de Guayaquil (BVG). Este volumen de transacciones contiene los valores efectivos (valor nominal por su precio) de las negociaciones 


\section{Activos Financieros administrados por el Instituto Ecuatoriano de Seguridad Social}

Vol. 2, núm. 1., (2018)

Elio Edwin Sánchez Suárez; Benita del Carmen Cañizalez de Aguirre

totales. El volumen total de las operaciones de valores negociados, durante el periodo de análisis, se resume de la siguiente manera en el presente cuadro:

Tabla 3: Volumen de Operaciones por Título Emitido por el IESS periodo 2007 - 2012 (en millones de dólares)

\begin{tabular}{|l|r|c|}
\hline PERIODO 2007-2012 & Efectivo & \multicolumn{1}{c|}{$\%$} \\
\hline Valores & 14,04 & $0,43 \%$ \\
\hline ACCIONES & $1.604,53$ & $49,48 \%$ \\
\hline BONOS DEL ESTADO & 528,52 & $16,30 \%$ \\
\hline CERT. DEPOSITO A PLAZO & 25,35 & $0,78 \%$ \\
\hline CERT. TESORERIA & 123,50 & $3,81 \%$ \\
\hline OBLIGACIONES & 41,43 & $1,28 \%$ \\
\hline PAPEL COMERCIAL & 93,86 & $2,89 \%$ \\
\hline PÓLIZAS DE ACUMULACIÓN & 227,50 & $7,02 \%$ \\
\hline CERTIFICADO DE INVERSIÓN & 68,11 & $2,10 \%$ \\
\hline REPORTO & 515,72 & $15,90 \%$ \\
\hline VALORES DE TITULARIZACIÓN & $\mathbf{3 . 2 4 2 , 5 6}$ & $\mathbf{1 0 0 , 0 0 \%}$ \\
\hline Total por título emitido & &
\end{tabular}

Fuente: Base de Datos Bolsa de Valores de Guayaquil

Durante el periodo 2007-2012, el efectivo del total de títulos emitidos fue de 3.242,56 millones de dólares que representa el $100 \%$ del total del volumen de operaciones. En cuanto a las acciones emitidas por el IESS en el Mercado de Valores fue de 14,04 millones de dólares que representa el $0,43 \%$ del total del volumen de operaciones.

En cuanto a operaciones en reporto, es decir, (crédito con garantía en títulos valores), fue de 68,11 millones de dólares que representa el 2,10\%, del total del volumen de operaciones. En 


\section{Activos Financieros administrados por el Instituto Ecuatoriano de Seguridad}

Social

Vol. 2, núm. 1., (2018)

Elio Edwin Sánchez Suárez; Benita del Carmen Cañizalez de Aguirre cuanto a las operaciones en pólizas de acumulación fue de 93,86 millones de dólares que representa el $2.89 \%$ del total del volumen de operaciones.

El volumen de operaciones en efectivo de obligaciones fue de 123,50 millones de dólares que representa el 3,81\% del total del volumen de operaciones. El volumen de operaciones en efectivo de los certificados de tesorería fue de 25,35 millones de dólares que representa el 0,78\% del total del volumen de operaciones.

El volumen de operaciones en efectivo de bonos del estado fue de 1.604,53 millones de dólares que representa el 49,48\%, del total del volumen de operaciones. El volumen de operaciones en efectivo de certificados de depósito a plazo fue de 528,52 millones de dólares que representa el 16,20\%, del total del volumen de operaciones.

El volumen de operaciones en efectivo de titularizaciones fue de 515,72 millones de dólares que representa el 15,90\% del total del volumen de operaciones. El volumen de operaciones en efectivo de papel comercial fue de 41,43 millones de dólares que representa el $1,28 \%$ del total del volumen de operaciones.

El volumen total de las operaciones de valores negociados, durante el periodo de análisis, se resume en el siguiente cuadro: 


\section{Activos Financieros administrados por el Instituto Ecuatoriano de Seguridad Social}

Vol. 2, núm. 1., (2018)

Elio Edwin Sánchez Suárez; Benita del Carmen Cañizalez de Aguirre

Tabla 4: Volumen de operaciones del IESS periodo 2007 - 2012 (en millones de dólares)

\begin{tabular}{|l|c|c|}
\hline PERIODO 2007 - 2012 & Efectivo & $\%$ \\
\hline Valor & 754,86 & $23 \%$ \\
Total por título emitido 2007 & 878,09 & $27 \%$ \\
Total por título emitido 2008 & 728,66 & $22 \%$ \\
\hline Total por título emitido 2009 & 587,50 & $18 \%$ \\
\hline Total por título emitido 2010 & 101,66 & $3 \%$ \\
Total por título emitido 2011 & 191,79 & $6 \%$ \\
Total por título emitido 2012 & $3.242,56$ & $100 \%$ \\
\hline TOTAL DEL PERIODO & & \\
\hline
\end{tabular}

Fuente: Base de datos Bolsa de Valores de Guayaquil

El total de activos financieros negociados en el 2007 suma 754,86 millones de dólares. El total de activos financieros negociados en el 2008 sumaron 878,09 millones de dólares. El total de activos financieros negociados en el 2009 sumaron 728,66 millones de dólares.

El total de activos financieros negociados en el 2010 sumaron 587,50 millones de dólares. El total de activos financieros negociados en el 2011 sumaron 101,66 millones de dólares. El total de activos financieros negociados en el 2012 sumaron 191,79 millones de dólares. 


\section{Activos Financieros administrados por el Instituto Ecuatoriano de Seguridad}

Social

Vol. 2, núm. 1., (2018)

Elio Edwin Sánchez Suárez; Benita del Carmen Cañizalez de Aguirre

Grafico 1: Volumen de operaciones del IESS periodo 2007-2012 (en millones de dólares)

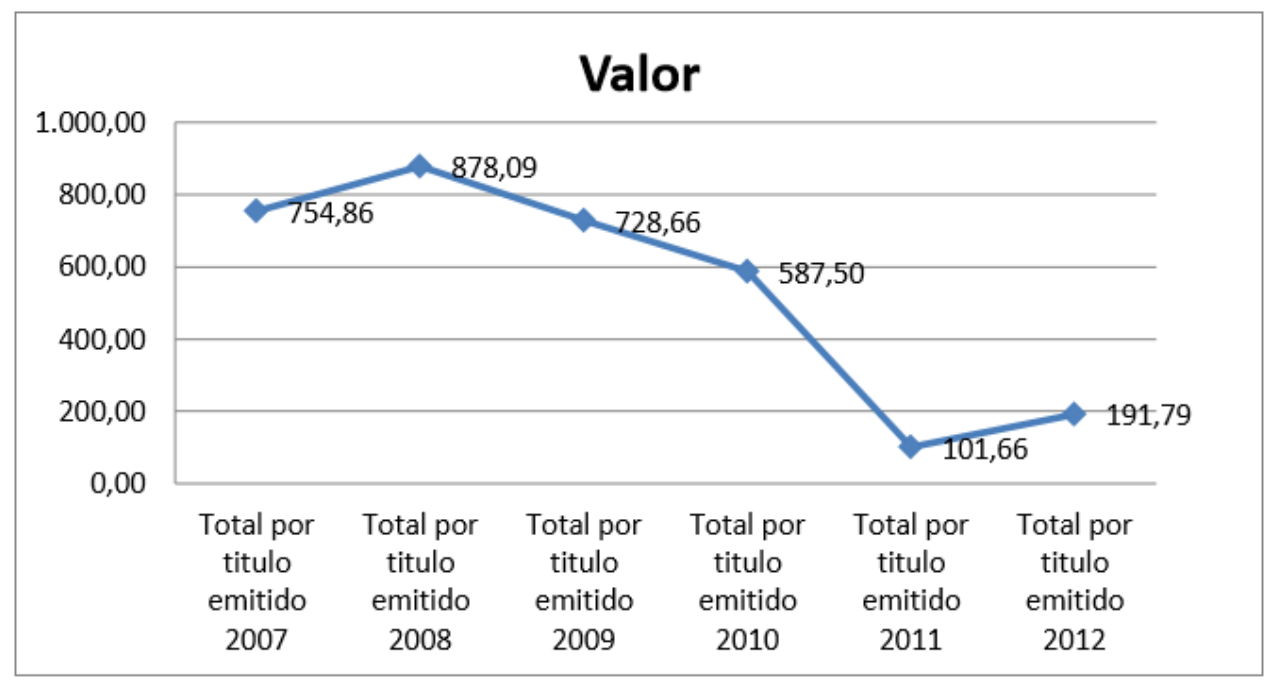

Fuente: Base de datos Bolsa de Valores de Guayaquil

Grafico 2: Tasas de interés período 2007-2012 (porcentaje)

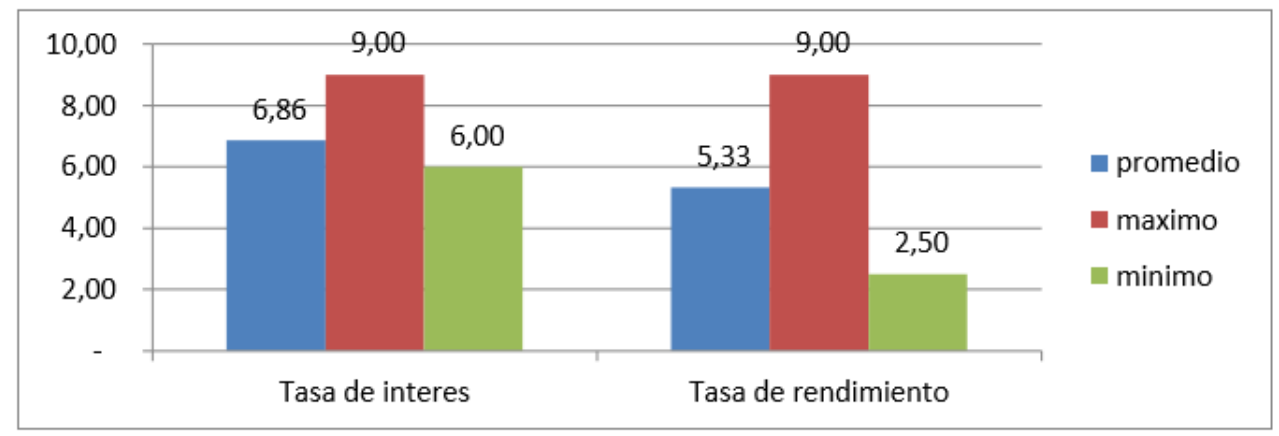

Fuente: Base de datos Bolsa de Valores de Guayaquil 


\section{Activos Financieros administrados por el Instituto Ecuatoriano de Seguridad Social}

Vol. 2, núm. 1., (2018)

Elio Edwin Sánchez Suárez; Benita del Carmen Cañizalez de Aguirre

Los títulos descritos en el apartado anterior están sujetos a tasas de interés y tasas de rendimiento, en promedio las tasas de interés en el periodo 2007-20112 fue de 6,86\% y las tasas de rendimiento de 5,33\%.

\section{Conclusiones.}

El estudio demuestra que el incremento de las inversiones del Instituto Ecuatoriano de Seguridad Social, ha provocado un aumento en su participación en el mercado de valores ecuatoriano durante el régimen del gobierno actual incrementando el consumo y la formación bruta de capital en el país, en este sentido, sabemos que las inversiones realizadas por el IESS han consistido en la adquisición de recursos en títulos valores de renta fija y renta variable de empresas del sector público y privado, que ofrezcan expectativas de seguridad, liquidez y rendimiento.

Son precisamente estas inversiones las que han dinamizado el sistema financiero y el mercado de valores ecuatoriano, lo que pronostican indicadores macroeconómicos bastante halagadores, así como el incremento del PIB en los sectores de intermediación financiera y la construcción.

Las inversiones del IESS. a través de su banco (BIESS) han aportado en el crecimiento del consumo de los hogares y del sector de la construcción, que son los sectores que más han dinamizado la economía ecuatoriana en los últimos años. Estas inversiones se han constituido como complemento a la liquidez que ha introducido sistemáticamente el gobierno por medio del gasto público. 


\section{Activos Financieros administrados por el Instituto Ecuatoriano de Seguridad}

Social

Vol. 2, núm. 1., (2018)

Elio Edwin Sánchez Suárez; Benita del Carmen Cañizalez de Aguirre

El Instituto Ecuatoriano de Seguridad Social IESS. Desde su institucionalización y fundación Siempre ha sido protagonista en el desarrollo del Ecuador pero es a partir de la vigencia de la Constitución del 2008 cuando el protagonismo antes mencionado se vuelve decisivo en el crecimiento sostenido de la economía ecuatoriana, ya que integra de manera estructural a la Seguridad Social a la Nueva Arquitectura Financiera en el Ecuador y por medio de esta a la Nueva Arquitectura Financiera Regional, el Modelo y Política Económica del Gobierno actual debe mantenerse para que finalmente se consolide un crecimiento económico fundamentado en la economía real y que tenga como fin último al Ser Humano.

Es indudable que la intervención del IESS. En el sector Financiero ecuatoriano ha sido positiva porque ha regulado el mercado monetario y financiero en el Ecuador, sobre todo a través de operaciones bursátiles en el mercado de valores, evidenciadas sustancialmente en el presente trabajo investigativo en el periodo 2007 - 2012, canalizando los recursos financiaros de los agentes excedentarios a actividades productivas materializadas y promovidas por agentes deficitarios de estos mismos recursos financieros, produciendo un efecto multiplicador en la economía ecuatoriana que atrae sistemáticamente mayor inversión tanto pública como privada, se recomienda que la intervención del Instituto Ecuatoriano de Seguridad Social IESS. se mantenga en el tiempo como parte sustancial de una Política Fiscal que conlleve a un crecimiento económico saludable. 


\section{Activos Financieros administrados por el Instituto Ecuatoriano de Seguridad Social}

Vol. 2, núm. 1., (2018)

Elio Edwin Sánchez Suárez; Benita del Carmen Cañizalez de Aguirre

\section{Bibliografía}

Asamblea Constituyente (2008) Constitución de la República del Ecuador. Quito.

Asamblea Constituyente (2006). Ley del Mercado de Valores. Quito.

Asamblea Constituyente (2005). Ley de la Seguridad Social. Quito.

Departamento Nacional De Estadísticas (1993) Manual de Normas y Procedimientos del Sistema de Información. Editorial IESS, Quito.

El Asegurado (2003), Revista informativa del IESS, octubre.

Hold, G., \& Szalachman, R. (1990). Ahorro y asignación de recursos financieros

Instituto Ecuatoriano De Estadísticas Y Censos (2010) Anuario de Estadísticas. Quito.

Instituto Ecuatoriano De Seguridad Social (2012). Recuperado el 25 de Agosto del 2013 en: http://www.biess.fin.ec/leyde-transparencia/resumen-de-inversiones

Organización Mundial Del Trabajo (1991) “Administración de la seguridad social”. Naciones Unidas.

Ross S., Randolph W. Wester F., Jeffrey F. (2006). Finanzas corporativas, Quinta Edición. Editorial Mc Graw Hill.

Secretaria Nacional De Planificación (2009). Plan Nacional de Desarrollo del Buen Vivir (200920013). Quito. 